\title{
Acute Respiratory Distress Syndrome Subphenotypes Respond Differently to Randomized Fluid Management Strategy
}

\author{
Katie R. Famous ${ }^{1}$, Kevin Delucchi ${ }^{2}$, Lorraine B. Ware ${ }^{3,4}$, Kirsten N. Kangelaris ${ }^{5}$, Kathleen D. Liu ${ }^{6,7}$, \\ B. Taylor Thompson ${ }^{8}$, and Carolyn S. Calfee ${ }^{1,7}$; for the ARDS Network \\ ${ }^{1}$ Division of Pulmonary and Critical Care Medicine, Department of Medicine, ${ }^{2}$ Department of Psychiatry, ${ }^{5}$ Division of Hospital \\ Medicine, Department of Medicine, ${ }^{6}$ Division of Nephrology, Department of Medicine, and ${ }^{7}$ Department of Anesthesia, University of \\ California San Francisco, San Francisco, California; ${ }^{3}$ Department of Medicine, and ${ }^{4}$ Department of Pathology, Microbiology, and \\ Immunology, Vanderbilt University, Nashville, Tennessee; and ${ }^{8}$ Division of Pulmonary and Critical Care, Department of Medicine, \\ Massachusetts General Hospital, Boston, Massachusetts
}

ORCID ID: 0000-0001-7460-0883 (K.R.F.)

\begin{abstract}
Rationale: We previously identified two acute respiratory distress syndrome (ARDS) subphenotypes in two separate randomized controlled trials with differential response to positive end-expiratory pressure.

Objectives: To identify these subphenotypes in a third ARDS cohort, to test whether subphenotypes respond differently to fluid management strategy, and to develop a practical model for subphenotype identification.

Methods: We used latent class analysis of baseline clinical and plasma biomarker data to identify subphenotypes in FACTT (Fluid and Catheter Treatment Trial; $\mathrm{n}=1,000)$. Logistic regression was used to test for an interaction between subphenotype and treatment for mortality. We used stepwise modeling to generate a model for subphenotype identification in FACTT and validated its accuracy in the two cohorts in which we previously identified ARDS subphenotypes.
\end{abstract}

Measurements and Main Results: We confirmed that a two-class (two-subphenotype) model best described the study population. Subphenotype 2 was again characterized by higher inflammatory biomarkers and hypotension. Fluid management strategy had significantly different effects on 90-day mortality in the two subphenotypes ( $P=0.0039$ for interaction); mortality in subphenotype 1 was $26 \%$ with fluid-conservative strategy versus $18 \%$ with fluid-liberal, whereas mortality in subphenotype 2 was $40 \%$ with fluid-conservative strategy versus $50 \%$ in fluid-liberal. A threevariable model of IL-8, bicarbonate, and tumor necrosis factor receptor-1 accurately classified the subphenotypes.

Conclusions: This analysis confirms the presence of two ARDS subphenotypes that can be accurately identified with a limited number of variables and that responded differently to randomly assigned fluid management. These findings support the presence of ARDS subtypes that may require different treatment approaches.

Keywords: subphenotype; acute lung injury; fluid therapy
The acute respiratory distress syndrome (ARDS) is a common and frequently fatal clinical condition characterized by disruption of the alveolar-capillary barrier with the formation of noncardiogenic, protein-rich pulmonary edema (1). Unfortunately, despite decades of research, treatment is limited to supportive care, such as low tidal-volume mechanical ventilation (2). In part, this failure to identify effective pharmacotherapies may be a result of the clinical and biologic heterogeneity within ARDS.

(Received in original form March 30, 2016; accepted in final form August 10, 2016)

Supported by National Institutes of Health grants T32 HL7185-39 and F32 HL129680-01 (K.R.F.), K23 HL116800 (K.N.K.), HL103836 and HL112656 (L.B.W.), HL110969 (C.S.C.), HL131621 (C.S.C.), and HL133390 (C.S.C.); and by National Institutes of Health/NHLBI ARDS Network contracts N01-HR 46046-64 and N01-HR 16146-54.

Author Contributions: K.R.F. drafted the manuscript. K.D. and K.R.F. performed the statistical analyses. K.N.K., K.D.L., and L.B.W. contributed biomarker measurements. C.S.C. supervised the study design, data interpretation, and manuscript preparation. K.R.F., K.D., L.B.W., K.N.K., K.D.L., B.T.T., and C.S.C. contributed significantly to study design and interpretation. All authors made significant intellectual contributions to the final manuscript and approved its submission.

Correspondence and requests for reprints should be addressed to Carolyn S. Calfee, M.D., M.A.S., 505 Parnassus Avenue, M1088, San Francisco, CA 941430111. E-mail: carolyn.calfee@ucsf.edu

This article has an online supplement, which is accessible from this issue's table of contents at www.atsjournals.org

Am J Respir Crit Care Med Vol 195, Iss 3, pp 331-338, Feb 1, 2017

Copyright $\odot 2017$ by the American Thoracic Society

Originally Published in Press as DOI: 10.1164/rccm.201603-0645OC on August 11, 2016

Internet address: www.atsjournals.org 


\section{At a Glance Commentary}

\section{Scientific Knowledge on the}

Subject: We recently identified two acute respiratory distress syndrome (ARDS) subphenotypes in two randomized controlled trial cohorts, with distinct natural histories, clinical and biomarker profiles, and differential response to therapy with positive endexpiratory pressure. It is unknown if ARDS subphenotypes respond differently to fluid-management strategy. Likewise, the optimal approach to efficient classification of ARDS subphenotypes is unknown.

\section{What This Study Adds to the}

Field: We report the novel finding that ARDS subphenotypes as identified by latent class analysis have a differential response to randomly assigned fluid management strategy. ARDS subphenotype could be identified with excellent accuracy using three variables in three distinct patient cohorts.

We recently identified two distinct ARDS subphenotypes in two independent studies (3). We used the statistical approach of mixture modeling, specifically latent class analysis (LCA), which uses multiple "class-defining" variables to estimate the optimal number of subclasses that best fit a sample. For those analyses, we used data from two randomized controlled trials (RCTs), ARMA and ALVEOLI (Assessment of Low tidal Volume and elevated Endexpiratory volume to Obviate Lung Injury) $(2,4)$. Remarkably, these separate datadriven analyses identified two very similar subphenotypes in each trial. Subphenotype 2 was associated with increased levels of inflammatory biomarkers, acidosis, and shock and was present in similar frequency in both ARMA and ALVEOLI. Importantly, although there was no overall mortality benefit to either positive endexpiratory pressure strategy in the primary analysis of the ALVEOLI trial, higher positive end-expiratory pressure was beneficial in one subphenotype and harmful in the other $(3,4)$. This statistically significant qualitative interaction between subphenotype and treatment, in which the treatment responses of the two subphenotypes are in opposite directions, suggests differing underlying pathophysiologic mechanisms.

The current analysis was designed to further our understanding of ARDS pathogenesis and subphenotypes. We hypothesized that ARDS subphenotypes would be present in a third, more recent independent cohort, the NHLBI ARDS Network's FACTT (Fluid and Catheter Treatment Trial), as they were in our prior analyses of the ARMA and ALVEOLI trials. We also hypothesized that these two subphenotypes would respond differently to randomly assigned fluid therapy. In our previous analysis, subphenotype 2 was characterized by higher levels of inflammatory cytokines and higher levels of both intercellular adhesion molecule- 1 and von Willebrand factor, markers of endothelial cell injury $(5,6)$. Therefore, given that subphenotype 2 had evidence of more baseline inflammation and endothelial cell injury, features that would favor more extravascular fluid accumulation, we hypothesized a priori that subphenotype 2 would have higher mortality and fewer ventilator-free days in response to a liberal fluid strategy. In addition, with the goal of defining a clinically applicable model for subphenotype identification at the bedside, we tested the optimal number and composition of variables that could be used to identify subphenotypes in FACTT (the largest of the three cohorts) and then validated this model in ARMA and ALVEOLI. Some of the results of these studies have been previously reported in the form of an abstract (7).

\section{Methods}

\section{Patient Population}

Patients for this secondary analysis were drawn from FACTT. Full details of the trial have been published $(8,9)$. Briefly, FACTT enrolled 1,000 patients with ARDS between 2000 and 2005. Patients were eligible if they met ARDS criteria within 48 hours before enrollment. The trial randomized subjects in a two-by-two factorial design; one arm compared conservative versus liberal fluid management, whereas the other arm compared monitoring patients with ARDS with a pulmonary artery versus central venous catheter. Clinical data and biologic samples for this analysis were obtained on study enrollment, before randomization. There was no difference in 60-day mortality with either intervention $(8,9)$. Patients assigned to the fluid-conservative group had significantly more ventilator-free days (8).

\section{Latent Class Analysis}

To estimate the optimal number of classes that best fit the FACTT cohort, we used LCA in a similar statistical approach as in our previous study $(10,11)$. Further detail on LCA can be found in the online supplement. We used similar baseline clinical and biomarker variables that were collected before treatment initiation (3). Clinical outcomes were not included. A full list of variables is available in the online supplement.

In our prior study, eight plasma biomarkers were incorporated into the latent class models; most of these biomarkers contributed substantially to distinguishing the two subphenotypes (3). We therefore included these biomarkers in the current analysis. Four biomarkers were measured previously for other studies (IL-6, IL-8, surfactant protein D, and von Willebrand factor) $(12,13)$; the other four were measured for this analysis (intercellular adhesion molecule-1, protein $\mathrm{C}$, soluble tumor necrosis factor receptor-1 [sTNFr1], and plasminogen activator inhibitor 1). We also included two previously measured ARDS biomarkers, angiopoietin-2 (Ang-2) and the receptor for advanced glycation end-products (RAGE), which were not available for our prior study $(12,13)$. All measurements for this analysis were conducted using ELISA. Additional assay details are available in the online supplement.

Variables were examined for their distributional properties, and continuous variables with marked skew were logtransformed. Race was collapsed into white/other because of a limited number of nonwhite patients. To estimate the model parameters, the continuous variables were placed on a $z$ scale with a mean of zero and SD of one. We used Mplus (version 7.4; Los Angeles, CA) to fit models with latent classes ranging from one to five classes. As in our previous study, to determine the best-fitting model, where the different models are defined by the number of classes, we used several indicators: (1) the Bayesian Information Criteria, where a decreasing number suggests improved 
Table 1. Model Fit Statistics for One to Five Latent Classes of Subjects with ARDS Enrolled in FACTT

\begin{tabular}{|c|c|c|c|c|c|c|c|c|}
\hline \multirow{2}{*}{$\begin{array}{l}\text { Number of } \\
\text { Classes }\end{array}$} & \multirow{2}{*}{$\begin{array}{l}\text { Bayesian } \\
\text { Information } \\
\text { Criterion }\end{array}$} & \multirow[b]{2}{*}{ Entropy } & \multicolumn{5}{|c|}{ Number of Individuals Assigned to Each Class or Subphenotype } & \multirow{2}{*}{$\begin{array}{c}P \text { Value, } \\
k \text { vs. } k-1 \\
\text { Classes }\end{array}$} \\
\hline & & & 1 & 2 & 3 & 4 & 5 & \\
\hline 1 & 93883.9 & - & 1,000 & & & & & - \\
\hline 2 & 92118.2 & 0.86 & 727 & 273 & & & & $<0.0001$ \\
\hline 3 & 91839.5 & 0.88 & 708 & 164 & 128 & & & 0.19 \\
\hline 4 & 91519.8 & 0.82 & 434 & 351 & 159 & 56 & & 0.66 \\
\hline 5 & 91267.7 & 0.84 & 411 & 287 & 157 & 92 & 53 & 0.08 \\
\hline
\end{tabular}

Definition of abbreviations: ARDS = acute respiratory distress syndrome; FACTT $=$ Fluid and Catheter Treatment Trial.

$P$ value represents the Vuong-Lo-Mendell-Rubin test, which tests if $k$ classes is a better model fit than $k-1$ classes.

model fit; (2) the Vuong-Lo-Mendell-Rubin test, which tests if $k$ classes is a better model fit than $k-1$ classes; and (3) the number of patients assigned to the smallest class, where a model with a very small class size would not be meaningful (3). We also examined entropy (indicating class separation) and the average probability of class assignment.

Once the number of classes was established, we used Student's $t$ tests, Pearson chi-square, or Wilcoxon rank sum to test for differences among classes. We analyzed the association between class assignment and clinical outcomes (60-d mortality, 90-d mortality, and ventilator-free days).

To test for a differential effect of treatment on clinical outcomes based on class assignment, we used a logistic regression model incorporating an interaction term for the product of class assignment and mortality; a negative-binomial regression model was used for the outcome of ventilator-free days. To assess if any differential effect of treatment was caused by severity of illness, we used logistic regression incorporating an interaction term for the product of the Acute Physiology and Chronic Health Evaluation III score and treatment.

We also performed a post hoc analysis comparing baseline hemodynamic measurements from central venous or pulmonary artery catheters between subphenotypes. These variables were not included in the LCA because they are not routinely measured in other ARDS studies, and their contribution to subphenotype classification would be difficult to validate outside of FACTT.

\section{Subphenotype Classification Model}

We next tested how well a smaller number of variables derived from FACTT could identify subphenotypes. We tested models with up to five variables, chosen because they contributed most significantly to class assignment in FACTT as identified using forward stepwise modeling. We also tested a model that only incorporated the topperforming variables available in routine clinical practice. Subjects were excluded if they did not have all of the variables incorporated in the classifier models. We tested the performance characteristics of these models using ARMA and ALVEOLI as validation cohorts because we had previously developed latent class models in these cohorts to use as a gold standard (3).

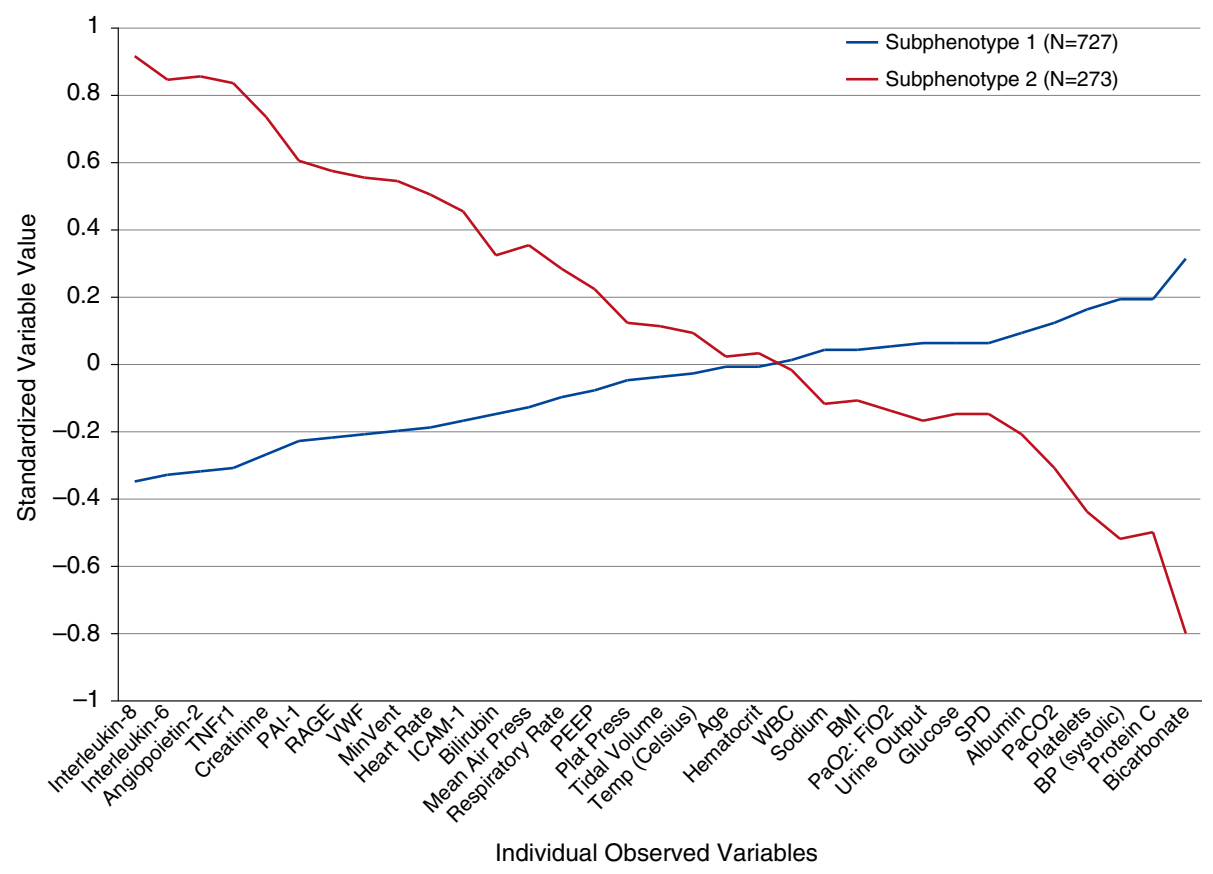

Figure 1. Continuous variables by subphenotype used in the latent class analysis of subjects enrolled in the Fluid and Catheter Treatment Trial. Individual continuous variables were placed on a $z$ scale with a mean of zero and SD of one. Standardized variable values for each subphenotype represent their variation from the cohort as a whole (i.e., a standardized variable value of +0.6 in subphenotype 2 reflects that the mean variable value in subphenotype 2 was 0.6 SD above the mean in the overall cohort). Variables are presented from left to right in order of maximum separation between subphenotypes 1 and 2. On the left side of the graph, standardized variables are higher in subphenotype 2; on the right side of the graph, standardized variables are lower in subphenotype 2. $\mathrm{BMI}=$ body mass index; BP (systolic) = systolic blood pressure; ICAM-1 = intercellular adhesion molecule-1; Mean Air Press = mean airway pressure; MinVent = total minute ventilation; PAl-1 = plasminogen activator inhibitor-1; PEEP = positive end-expiratory pressure; Plat Press = plateau pressure; RAGE = receptor for advanced glycation end-products; SPD = surfactant protein D; Temp (Celsius) = temperature in degrees Celsius; TNFr1 = tumor necrosis factor receptor -1 ; $V W F=$ von Willebrand factor; $\mathrm{WBC}=$ white blood cell count. 
We did not include Ang-2 or RAGE in these models, because these biomarkers were not measured in ARMA and ALVEOLI. Full details of these trials are provided in the original publications and in our prior publication (2-4). Lastly, to test if we could detect a differential effect of treatment when subphenotype is defined by a limited-variable model rather than the full LCA, we used a logistic regression model that incorporated an interaction term for the product of class assignment as defined by the limited-variable model and mortality. Because the classifier model is logistic regression-based, subjects with missing data were excluded.

\section{Results}

\section{A Two-Class Model Best Describes the Patients with ARDS Enrolled in FACTT}

We began by fitting latent class models ranging from one to five classes. The Bayesian Information Criteria decreased as the number of classes increased, suggesting that the addition of subsequent classes may be adding additional information to the model (Table 1). Entropy in all models was greater than 0.80 , indicating strong separation between the classes. Using the Vuong-Lo-Mendell-Rubin test, a two-class model was a significant improvement over a one-class model $(P<0.0001)$; additional classes did not provide a statistically significant improvement. We retained a final two-class model based on these results.

The two-class model estimated 727 subjects in class 1 and 273 subjects in class 2 . The average latent class probabilities were 0.97 for class 1 and 0.93 for class 2, indicating robust probability of class assignment. These findings were similar to our previous analysis (3). For subsequent analyses, subjects were assigned to their most likely class. Because of the high average latent class probabilities of class assignment, there is minimal loss of information in this approach. We subsequently refer to class 1 and 2 as subphenotypes 1 and 2, as in our previous analysis (3).

\section{ARDS Subphenotypes Have Distinct Characteristics and Clinical Outcomes}

The baseline clinical and biologic characteristics of the two subphenotypes were similar to our prior work (see Table
E1 in the online supplement) (3). Subphenotype 2 had higher levels of IL-8, IL-6, and sTNFr1, but lower serum bicarbonate and protein C (Figure 1; see Table E2). Subphenotype 2 also had higher plasma levels of Ang-2 and RAGE, which were newly incorporated into this analysis. There was no significant difference in sex distribution between the subphenotypes $(P=0.38)$ (Figure 2). Subphenotype 1 had a higher proportion of white patients (66 vs. $58 \%$; $P=0.02$ ) (Figure 2). As in our prior analyses, patients in subphenotype 2 more frequently required vasopressors at study enrollment (68\% vs. $20 \%$; $P<0.0001)$ (Figure 2).

Consistent with our prior work, there was a significant difference in primary ARDS risk factor by subphenotype $(P<$ 0.0001 ) (3). As in our prior studies, a greater proportion of subjects in subphenotype 2 had sepsis as their primary ARDS risk factor than in subphenotype 1 (see Figure E1); however, subjects with sepsis as their primary ARDS risk factor were equally likely to be assigned to either subphenotype (49\% subphenotype 1; 51\% subphenotype 2). Subjects with trauma, aspiration, or pneumonia as their primary risk factors were more likely to be assigned to subphenotype 1 (Figure 3; see Figure E1). Subjects in subphenotype 2 had a higher central venous pressure (12.7 vs. $11.7 \mathrm{~mm}$ $\mathrm{Hg} ; \mathrm{P}=0.005)$ and lower central venous

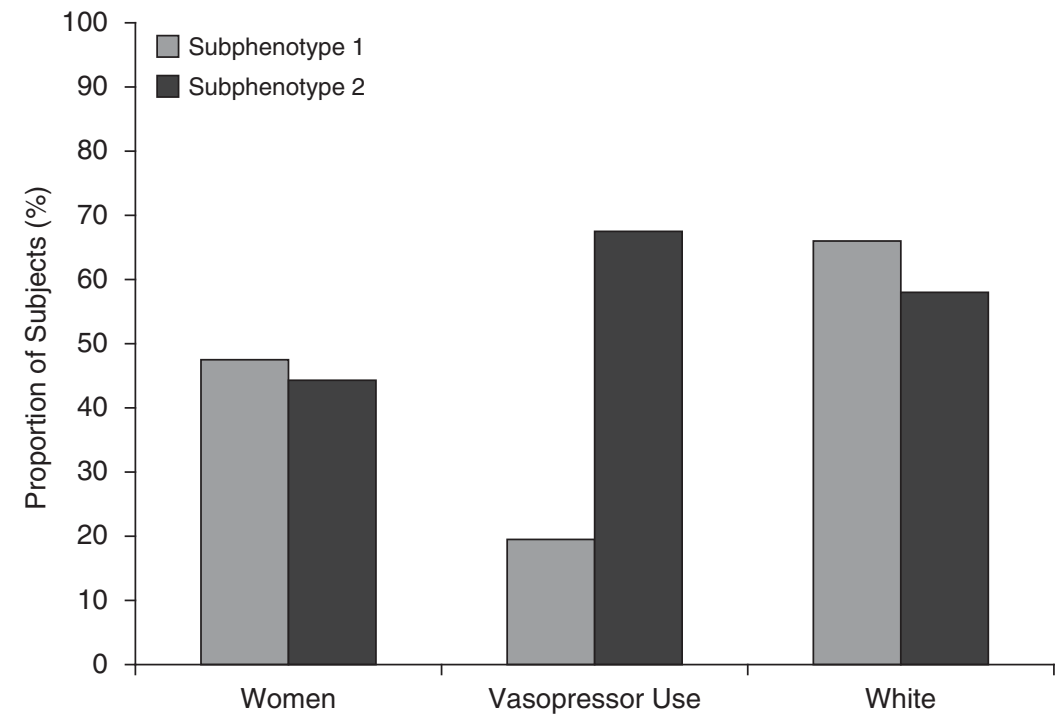

Figure 2. Categorical variables by subphenotype. There was no significant difference in sex distribution between the two subphenotypes $(P=0.38)$. Subphenotype 1 had a higher proportion of white patients $(P=0.02)$. Subphenotype 2 more frequently required vasopressors at study enrollment $(P<0.0001)$. Chi-squared analyses were performed for all comparisons. 


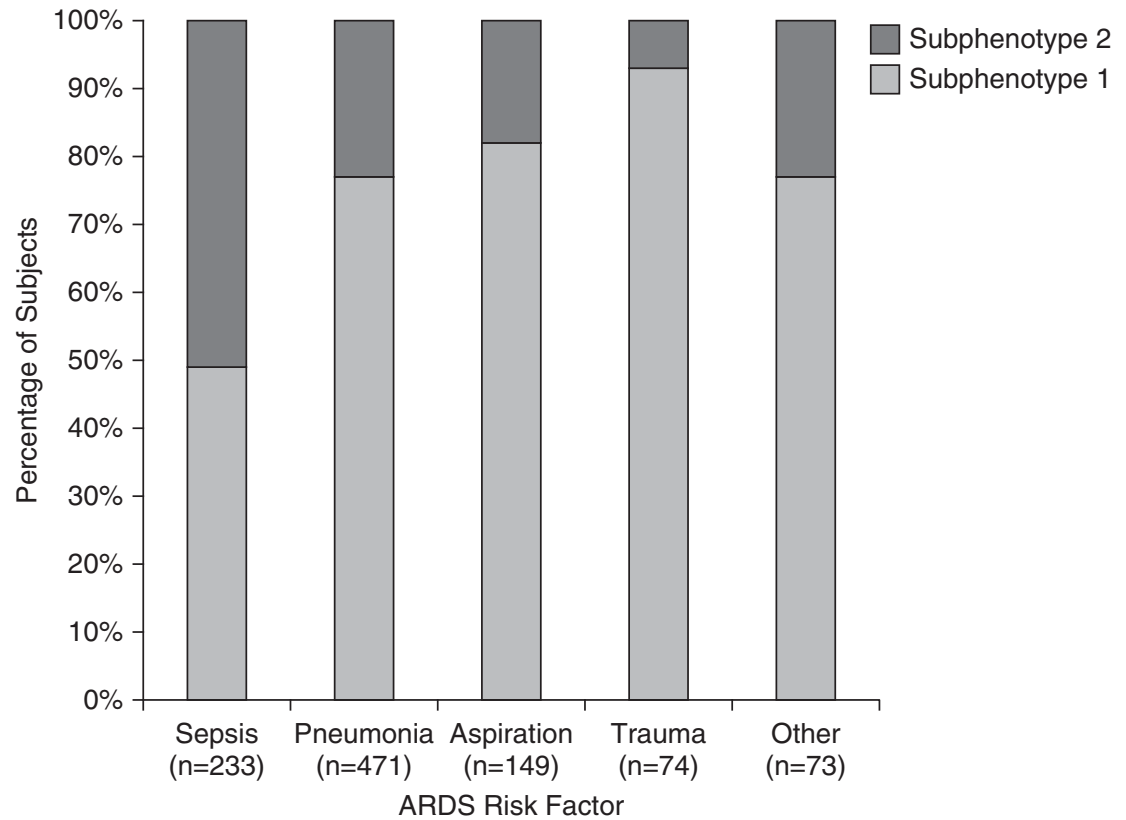

Figure 3. Within each acute respiratory distress syndrome (ARDS) risk factor, the proportion of subjects assigned to each subphenotype.

There was no significant interaction between subphenotype and catheter type for 60- or 90-day mortality or for ventilator-free days (see Table E3). In addition, there was no significant interaction between Acute Physiology and Chronic Health Evaluation III score and fluid-management strategy for 60 - or 90 -day mortality ( $P=0.42$ and 0.25 , respectively).

\section{A Three-Variable Model Can Accurately Identify ARDS Subphenotype}

Using forward stepwise modeling, the five variables that contributed most to subphenotype assignment in FACTT (in order of contribution) were IL-8, serum bicarbonate, sTNFr1, vasopressor use, and total minute ventilation. We then measured the accuracy of models using the top three to five of these variables for subphenotype identification in the three patient cohorts (Table 5). The gold standard for subphenotype was assignment by LCA (3). The top three variable model had an area under the receiver operating characteristic curve (AUC) of 0.95 in FACTT, 0.94 in ARMA, and 0.91 in ALVEOLI. Using the Youden index, the optimal sensitivity and specificity of the three-variable model derived from FACTT were $87 \%$ and $93 \%$, respectively. This three-variable model identified subphenotype 1 with greater accuracy as compared with subphenotype 2 in all three cohorts (see Tables E4-E6). There was no consistent improvement in AUC with a four- or five-variable model (Table 5). The parameter estimates for the three-variable model are listed in Table 6.

Because IL- 8 and sTNFr1 are not available in routine clinical care, we also tested the accuracy of a model that only incorporates clinically available variables to accurately identify subphenotype. In order of contribution, the clinical variables that contributed most to subphenotype identification in FACTT were bicarbonate, vasopressor use, creatinine, total minute ventilation, heart rate, primary ARDS risk factor, and systolic blood pressure. This clinical variables-only model had an AUC of 0.93 in FACTT. Using the Youden index, the optimal sensitivity and specificity of this model were both $84 \%$. When this model was applied to the validation cohorts, it had AUCs of 0.75 and 0.80 in ARMA and ALVEOLI, respectively.

To further evaluate the utility of the three-variable model, we used logistic regression to determine whether we could detect the interaction between treatment and subphenotype as identified using this model $(n=859)$. Although the same direction of differential treatment effect was observed in this analysis, the interaction was not statistically significant (see Table E7). We performed exploratory analyses to assess for differences in mortality, severity of illness, demographics, or other variables that contributed to class separation between those subjects with missing versus complete data. Subjects excluded from this analysis by virtue of missing data were more likely to be nonwhite than those with complete data $(P=0.049)$ and have higher protein $C$ $(P=0.01)$ and arterial carbon dioxide levels $(P=0.009)$ (see Table E8).

\section{Discussion}

We have now identified two ARDS subphenotypes in three separate clinical trials, with remarkable similarity in their characteristics and outcomes across these trials (3). We discovered a qualitative interaction for mortality between ARDS subphenotype as defined by LCA and fluidmanagement strategy, in which a fluid-

Table 2. Baseline Hemodynamic Measurements by ARDS Subphenotype

\section{Subphenotype 1}

Central venous pressure, $\mathrm{mm} \mathrm{Hg}$

Pulmonary artery occlusion pressure, $\mathrm{mm} \mathrm{Hg}$

Central venous oxygen, \%

Mixed venous oxygen saturation, \%

Cardiac index, $\mathrm{L} / \mathrm{min} / \mathrm{m}^{2}$
$11.7 \pm 4.7$
$15.5 \pm 5.4$
$73.5 \pm 10.8$
$69.2 \pm 11.4$
$4.3 \pm 1.4$

Subphenotype 2

$12.7 \pm 4.7$
$16.1 \pm 5.3$
$71.4 \pm 11.9$
$67.8 \pm 12.6$
$4.1 \pm 1.4$

Definition of abbreviation: ARDS = acute respiratory distress syndrome. 
Table 3. Clinical Outcomes by ARDS Subphenotype

\begin{tabular}{lccc}
\hline & $\begin{array}{c}\text { Subphenotype 1 } \\
(\boldsymbol{n}=\mathbf{7 2 7})\end{array}$ & $\begin{array}{c}\text { Subphenotype 2 } \\
(\boldsymbol{n}=\mathbf{2 7 3 )}\end{array}$ & $\boldsymbol{P}$ Value \\
& 21 & 44 & $<0.0001$ \\
60-d mortality, \% & 22 & 45 & $<0.0001$ \\
90-d mortality, \% & 19 & 3 & $<0.0001$ \\
Ventilator-free days, median & & & \\
\hline
\end{tabular}

Definition of abbreviation: ARDS = acute respiratory distress syndrome.

$P$ value represents chi-square analysis for mortality and Wilcoxon rank sum for ventilator-free days.

conservative strategy was associated with improved mortality in subphenotype 2 but had the opposite effect in subphenotype 1. Also, although we previously reported that a limited number of variables (IL-6, sTNFr1, and vasopressor use) could accurately identify subphenotype, we sought to optimize an identification model for potential use at the bedside (3). In this study, we found that a three-variable model that incorporates IL-8, serum bicarbonate, and TNFr1 identifies ARDS subphenotypes with better accuracy than a model that only incorporates clinically available variables.

Although it would have been possible to use a limited-variable model derived from ARMA or ALVEOLI to assign subphenotype in FACTT, we performed a full LCA in FACTT for two reasons. First, because FACTT is the largest ARDS Network trial, and because larger derivation cohorts generally lead to improved model performance, we thought FACTT would be the optimal cohort in which to build our limited-variable model for ARDS subphenotype classification. Second, we wanted to determine if we would detect similar subphenotypes in a larger, more recent cohort of patients with ARDS that reflects more contemporary management of ARDS and critical illness.

Although the subphenotypes had many similarities to those described in our prior analysis, we identified two notable differences. First, Ang-2 and RAGE, which were not available in our previous study, provide additional information about the underlying biology that distinguishes the two subphenotypes (3). Ang-2 is a biomarker and mediator of endothelial cell injury, whereas RAGE has been implicated as a marker of lung epithelial cell injury and inflammation $(12,14-16)$. Both plasma Ang-2 and RAGE levels were higher in subjects assigned to subphenotype 2, suggesting a higher degree of baseline endothelial and lung epithelial cell injury in these patients. Of note, a sensitivity analysis excluding Ang-2 and RAGE from the LCA models did not substantively alter the results (data not shown). Second, baseline creatinine was higher in subphenotype 2 compared with subphenotype 1 in the current analysis, whereas it did not contribute notably to class separation in ARMA or ALVEOLI (3). This difference may have been caused by differences in time to enrollment from ARDS development (36 $\mathrm{h}$ in ARMA and ALVEOLI vs. $48 \mathrm{~h}$ in FACTT) (8).

The original FACTT analysis did not find a mortality difference between subjects randomized to a fluid-liberal versus a fluidconservative approach, although those subjects randomized to fluid-conservative therapy had more ventilator-free days (8).
As a result, a fluid-conservative approach has been recommended for all patients with ARDS once shock has resolved (17). Our findings, however, suggest that there may be heterogeneity of treatment effects of fluid-management strategy in ARDS (18). Subjects in subphenotype 2 had lower mortality when randomized to a conservative strategy, whereas subjects in subphenotype 1 had higher mortality when randomized to the same approach. The mechanisms underlying this difference remain speculative. The higher Ang-2 levels in subphenotype 2 may reflect more endothelial permeability and predilection for extravascular fluid accumulation that responds favorably to fluid restriction, whereas fluid restriction in subphenotype 1 may lead to decreased intravascular volume, oxygen delivery, and perfusion.

Although patients in subphenotype 2 had fewer ventilator-free days than those in subphenotype 1 , there was not a significant interaction between fluid-management strategy and subphenotype for this outcome, in contrast to the analyses of mortality. This discrepancy suggests that the interaction between subphenotype and fluid-strategy on mortality may be driven by the extrapulmonary manifestations of the patient's underlying illness. There was not a clinically meaningful difference in baseline central venous pressure or cardiac index between subphenotypes, suggesting that the differential response to fluid management is probably not caused by differences in baseline hemodynamics.

We have concluded that we are observing similar subphenotypes across ARMA, ALVEOLI, and FACTT for a few reasons. First, a two-class model best fit the data in all three cohorts; these two subphenotypes have remarkably similar prevalence across the cohorts, and similar natural histories and clinical outcomes.

Table 4. Interaction between ARDS Subphenotype and Fluid-Management Strategy for the Outcomes of Mortality and Ventilator-Free Days

Subphenotype 1

\section{Fluid-management strategy}

Liberal $(n=367)$
Subphenotype 2

\begin{tabular}{ccl}
\hline Conservative $(\boldsymbol{n}=\mathbf{1 4 2})$ & Liberal $(\boldsymbol{n}=\mathbf{1 3 1})$ & $\boldsymbol{P}$ Value \\
& & \\
39 & 49 & 0.0093 \\
40 & 50 & 0.0039 \\
5 & 0 & 0.35 \\
\hline
\end{tabular}

60-d mortality, \%

90-d mortality, \%

Ventilator-free days, median

$\begin{array}{ll}24 & 17 \\ 26 & 18 \\ 17 & 21\end{array}$

$\begin{array}{rr}17 & 39 \\ 18 & 40 \\ 21 & 5\end{array}$

Definition of abbreviation: ARDS = acute respiratory distress syndrome.

$P$ value represents the interaction between subphenotype as defined by latent class analysis and randomly assigned fluid-management strategy for the outcome. 
Table 5. Validation of Limited-Variable Models

FACTT Derivation Cohort (AUC)
ARMA Validation

Cohort (AUC)
ALVEOLI Validation Cohort (AUC)

0.91

0.86

0.88

Three-variable model (IL-8, bicarbonate, TNFr1)

Four-variable model (IL-8, bicarbonate, TNFr1, vasopressor use)

Five-variable model (IL-8, bicarbonate, TNFr1,

0.95
0.97

0.97
0.94

0.89

0.90

vasopressor use, total minute ventilation)

Definition of abbreviations: ALVEOLI = Assessment of Low tidal Volume and elevated Endexpiratory volume to Obviate Lung Injury trial; AUC = area under the receiver operating characteristic curve; FACTT = Fluid and Catheter Treatment Trial; TNFr1 = tumor necrosis factor receptor-1.

Second, when we compare which variables contribute most to class separation, these variables are quite similar across the three studies (3). IL-6, IL-8, and TNFr1 were all among the top five continuous variables that were higher in subphenotype 2 as compared with subphenotype 1 in all three cohorts. Similarly, bicarbonate, protein C, systolic blood pressure, and platelets were all among the top five continuous variables that were lower in subphenotype 2 as compared with subphenotype 1 across the three cohorts. Third, a three-variable model derived from FACTT identifies subphenotype assignment in ARMA and ALVEOLI with a high degree of accuracy (AUC $>0.9$ in both validation cohorts), strongly suggesting that we are observing similar subphenotypes across the three trials with similar underlying biology. Using the limited-variable models to compare the AUC for correct subphenotype identification provides a quantitative assessment of the similarity of these subphenotypes across the three cohorts.

Although a major purpose of this study was to apply LCA to a third independent cohort to determine if this data-driven approach would identify similar subphenotypes in the FACTT trial that were identified in ARMA and ALVEOLI, future analyses are necessary to determine if these subphenotypes have differing underlying pathophysiologic mechanisms leading to lung injury and ARDS.

Furthermore, studies that incorporate additional clinical variables or plasma biomarkers in new populations of patients with ARDS may reveal that the hypoinflammatory or the hyperinflammatory groups encompass more than one subphenotype. Thus, this process of studying and measuring biologic markers and clinical factors to identify subphenotypes in ARDS is not just validation, but also discovery oriented, and may provide more insights into the pathogenesis of ARDS subphenotypes in future studies.

This study has several strengths. We used data from a trial of 1,000 patients, which represents a large sample size for ARDS clinical trials. In addition, FACTT was a RCT, which strengthens the ability to make causal inferences about differences between treatment groups. We also included measurements of 10 biomarkers associated with the pathogenesis and prognosis of ARDS, many of which were critical in separating the two subphenotypes.

This study also has important limitations. First, it is a secondary analysis and requires prospective validation before definitive conclusions regarding therapy can be drawn. Likewise, although the statistical test for interaction identifies that the two subphenotypes respond in opposite and significantly different directions to fluid management strategy, it does not specify whether the effect within either subphenotype is statistically significant. To prospectively test if subphenotypes respond differentially to randomized therapies, we first need to be able to identify these subjects at the bedside. This approach will likely require the development of rapid point-of- care biomarker assays, and then validation of a clinically feasible model to assign subphenotypes based on these biomarker assays. Second, it remains unknown if these subphenotypes exist outside RCTs in a more diverse population of critically ill patients. Testing for ARDS subphenotypes in more heterogeneous, non-RCT samples is an important future direction of this line of research.

Third, these analyses apply to variables obtained on study enrollment. Further research is needed to determine if these subphenotypes remain stable later in the course of ARDS. Finally, although a threevariable model had good accuracy in identifying subphenotypes in three separate study cohorts, when subphenotype was defined by the three-variable model rather than the full LCA, we were no longer able to detect the statistically significant differential response to fluid management by subphenotype. This difference is likely caused by missing data leading to decreased power to detect an interaction, and the modest amount of misclassification using the three-variable model; in addition, the excluded subjects with missing data may be different from subjects who were included with complete data, as suggested by the

Table 6. Logistic Regression Parameter Estimates for the Three-Variable Model in FACTT

\begin{tabular}{lrrrcr}
\hline Parameter & DF & Estimate & SE & Wald Chi-Square & $P$ Value \\
Intercept & 1 & 2.25 & 0.19 & 142.4 & $<0.0001$ \\
IL-8 & 1 & -1.97 & 0.20 & 99.6 & $<0.0001$ \\
Bicarbonate & 1 & 1.71 & 0.18 & 95.8 & $<0.0001$ \\
sTNFr1 & 1 & -1.71 & 0.19 & 83.5 & $<0.0001$ \\
\hline
\end{tabular}

Definition of abbreviations: DF = degrees of freedom; FACTT = Fluid and Catheter Treatment Trial; sTNFr1 = soluble tumor necrosis factor receptor-1.

The predictor variables for the logistic regression model are IL-8, bicarbonate, and sTNFr1. As in the latent class analysis, these variables were log-transformed and $z$ scaled. The outcome variable is class assignment. 
analyses in Table E8 (i.e., informative missingness). Unfortunately, LCA on its own cannot be used at the bedside to classify patients for clinical trials, so additional work is needed to further refine classification models to optimize their performance before clinical application.

In summary, this analysis supports the presence of two distinct subphenotypes of ARDS. Replication in three separate cohorts, along with the finding that these latent class-defined subphenotypes respond differentially to two randomly assigned treatments strongly supports the possibility that different pathophysiologic processes underlie class assignment (3). To advance the understanding of ARDS subphenotypes, a better understanding of the biologic mechanisms leading to ARDS in each subgroup is needed. Clinical application will require real-time assignment of ARDS subphenotype using a limited number of variables, a goal that requires the development of rapid biomarker assays and additional study of the optimal classifier variables. If it is possible to prospectively enroll patients in clinical trials by ARDS subphenotype, it may be possible to develop more targeted therapeutics for ARDS.

Author disclosures are available with the text of this article at www.atsjournals.org

Acknowledgment: The authors thank Dr. Michael Matthay for his contributions in support of this project.

\section{References}

1. Matthay MA, Ware LB, Zimmerman GA. The acute respiratory distress syndrome. J Clin Invest 2012;122:2731-2740.

2. The Acute Respiratory Distress Syndrome Network. Ventilation with lower tidal volumes as compared with traditional tidal volumes for acute lung injury and the acute respiratory distress syndrome. $N$ Engl $J$ Med 2000;342:1301-1308.

3. Calfee CS, Delucchi K, Parsons PE, Thompson BT, Ware LB, Matthay MA; NHLBI ARDS Network. Subphenotypes in acute respiratory distress syndrome: latent class analysis of data from two randomised controlled trials. Lancet Respir Med 2014;2:611-620.

4. Brower RG, Lanken PN, Maclntyre N, Matthay MA, Morris A, Ancukiewicz M, Schoenfeld D, Thompson BT; National Heart, Lung, and Blood Institute ARDS Clinical Trials Network. Higher versus lower positive end-expiratory pressures in patients with the acute respiratory distress syndrome. N Engl J Med 2004;351: 327-336.

5. Ware LB, Eisner MD, Thompson BT, Parsons PE, Matthay MA. Significance of von Willebrand factor in septic and nonseptic patients with acute lung injury. Am J Respir Crit Care Med 2004;170: 766-772.

6. Walter JM, Wilson J, Ware LB. Biomarkers in acute respiratory distress syndrome: from pathobiology to improving patient care. Expert Rev Respir Med 2014;8:573-586.

7. Famous KR, Delucchi K, Ware LB, Liu K, Kangelaris KN, Thompson BT, Matthay $M$, Calfee CS. Is a fluid-conservative strategy optimal for all patients with acute respiratory distress syndrome (ARDS)? Latent class analysis indicates that ARDS endotypes have a differential response to fluid-management strategy in the NHLBI ARDS Network Fluid and Catheter Treatment Trial (FACTT) [abstract]. Am J Respir Crit Care Med 2016;193:A6445.

8. Wiedemann HP, Wheeler AP, Bernard GR, Thompson BT, Hayden D, deBoisblanc B, Connors AF Jr, Hite RD, Harabin AL; National Heart, Lung, and Blood Institute Acute Respiratory Distress Syndrome (ARDS) Clinical Trials Network. Comparison of two fluidmanagement strategies in acute lung injury. N Engl J Med 2006;354: 2564-2575.
9. Wheeler AP, Bernard GR, Thompson BT, Schoenfeld D, Wiedemann HP deBoisblanc B, Connors AF Jr, Hite RD, Harabin AL; National Heart, Lung, and Blood Institute Acute Respiratory Distress Syndrome (ARDS) Clinical Trials Network. Pulmonary-artery versus central venous catheter to guide treatment of acute lung injury. $N$ Engl $J$ Med 2006;354:2213-2224.

10. Hagenaars JA, McCutcheon AL. Applied latent class analysis. New York: Cambridge University Press; 2002.

11. McLachlan G, Peel D. Finite mixture models. New York: John Wiley \& Sons, Inc; 2000.

12. Calfee CS, Gallagher D, Abbott J, Thompson BT, Matthay MA, Network NA; NHLBI ARDS Network. Plasma angiopoietin-2 in clinical acute lung injury: prognostic and pathogenetic significance. Crit Care Med 2012;40:1731-1737.

13. Calfee CS, Janz DR, Bernard GR, May AK, Kangelaris KN, Matthay MA, Ware LB; NIH NHLBI ARDS Network. Distinct molecular phenotypes of direct vs indirect ARDS in single-center and multicenter studies. Chest 2015;147:1539-1548.

14. Agrawal A, Matthay MA, Kangelaris KN, Stein J, Chu JC, Imp BM, Cortez A, Abbott J, Liu KD, Calfee CS. Plasma angiopoietin-2 predicts the onset of acute lung injury in critically ill patients. Am J Respir Crit Care Med 2013;187:736-742.

15. Bhandari V, Choo-Wing R, Lee CG, Zhu Z, Nedrelow JH, Chupp GL, Zhang X, Matthay MA, Ware LB, Homer RJ, et al. Hyperoxia causes angiopoietin 2-mediated acute lung injury and necrotic cell death. Nat Med 2006;12:1286-1293.

16. Briot R, Frank JA, Uchida T, Lee JW, Calfee CS, Matthay MA. Elevated levels of the receptor for advanced glycation end products, a marker of alveolar epithelial type I cell injury, predict impaired alveolar fluid clearance in isolated perfused human lungs. Chest 2009;135:269-275.

17. Wilson JG, Matthay MA. Mechanical ventilation in acute hypoxemic respiratory failure: a review of new strategies for the practicing hospitalist. $J$ Hosp Med 2014;9:469-475.

18. Iwashyna TJ, Burke JF, Sussman JB, Prescott HC, Hayward RA, Angus DC. Implications of heterogeneity of treatment effect for reporting and analysis of randomized trials in critical care. Am J Respir Crit Care Med 2015;192:1045-1051. 